Article

\title{
A Duality Result for the Generalized Erlang Risk Model
}

\section{Lanpeng $\mathrm{Ji}^{1, *}$ and Chunsheng Zhang ${ }^{2}$}

${ }^{1}$ Department of Actuarial Science, University of Lausanne, Bâtiment Extranef, UNIL-Dorigny, 1015 Lausanne, Switzerland

${ }^{2}$ School of Mathematical Sciences and LPMC, Nankai University, Tianjin 300071, China;

E-Mail: zhangcs@nankai.edu.cn

* Author to whom correspondence should be addressed; E-Mail: lanpeng.ji@ unil.ch;

Tel: +41-21-692-3376.

External Editor: Dr. Michael R. Powers

Received: 18 September 2014; in revised form: 21 October 2014 / Accepted: 27 October 2014 / Published: 6 November 2014

\begin{abstract}
In this article, we consider the generalized Erlang risk model and its dual model. By using a conditional measure-preserving correspondence between the two models, we derive an identity for two interesting conditional probabilities. Applications to the discounted joint density of the surplus prior to ruin and the deficit at ruin are also discussed.
\end{abstract}

Keywords: generalized Erlang risk model; duality; conditional measure-preservation; the Lundberg fundamental equation; joint density; surplus prior to ruin; deficit at ruin

\section{Introduction}

Since the publication of the seminal papers [1,2], there have appeared numerous contributions concerning different extensions of them. When reviewing these two papers, we found out that there are still some nice ideas that have not yet been extensively explored in the current insurance literature, for instance, the idea of measure-preservation, which played a key role in their derivation. Specifically, consider a compound Poisson risk model:

$$
Z(t)=Z(0)+c t-\sum_{i=1}^{N(t)} X_{i}, \quad t \geq 0
$$


where $Z(0) \geq 0$ is the deterministic initial reserve, $c>0$ is the rate of premium income and $\sum_{i=1}^{N(t)} X_{i}$ is a compound Poisson process representing the aggregated claim amounts up to time $t$. By introducing a dual model of the risk model $\{Z(t), t \geq 0\}$ and using the measure-preserving correspondence between them, Gerber and Shiu easily proved the generalized Dickson formula based on the following dual identity (1). Namely, for any $x>0$, if $c t \geq x$, then:

$$
\mathbb{P}(Z(s)>0, s \in(0, t) \mid Z(0)=0, Z(t)=x)=\mathbb{P}(Z(s)<x, s \in(0, t) \mid Z(0)=0, Z(t)=x) .
$$

The above dual identity and its variations turned out to be very important in insurance mathematics; see [3-8] and the references therein. Additionally, in [9] and [10], the idea of rotating the axis together with the measure-preservation makes it possible to transfer the study of the ruin time into the study of first passage time. This turns out to be helpful, since the study of first passage time is much easier for the upwards skip-free risk process. We refer to [11-14] and the references therein for other related applications of the measure-preservation.

As an important generalization of the compound Poisson risk model, the generalized Erlang risk model has also been extensively investigated in recent years; see [15,16] and the references therein. In this article, we aim to discuss various applications of the (conditional) measure-preservation in the generalized Erlang risk models; we refer to Section 3 for the precise definition of the (conditional) measure-preservation. Since, as is known, some risk models can be seen as "dual" of queueing models, we hope that the results in this paper will give some insight into more general studies.

The outline of the rest of the article is as follows: In Section 2, we introduce the generalized Erlang risk model and its dual model. Section 3 presents our main results. The principal result, Theorem 2, is concerned with the equivalence of a conditional probability related to the generalized Erlang risk model and a corresponding one related to the dual model. As an application of Theorem 2, we show in Theorem 3 our second result, which extends (1) for a certain Erlang risk model. In Section 4, we discuss some applications of the principal result to the calculation of the discounted joint density of the surplus prior to ruin and the deficit at ruin. In Theorem 4, we derive an expression for a crucial matrix function appearing in the discounted joint density, which could give a probabilistic explanation of the matrix function. We also provide a new proof for the known result on the discounted joint density. All of the proofs are shown in Section 5.

\section{Generalized Erlang Risk Model and Its Dual Model}

Let $V$ be a generalized $\operatorname{Erlang}(n, \boldsymbol{\lambda})$ distributed random variable with parameter $\boldsymbol{\lambda}=\left(\lambda_{1}, \cdots, \lambda_{n}\right)$, $\lambda_{i}>0, i \leq n$, i.e., $V=W_{1}+W_{2}+\cdots+W_{n}$, where $W_{i}, i \leq n$ are independent exponentials with parameters $\lambda_{i}, i \leq n$, respectively. It is known that the generalized $\operatorname{Erlang}(n, \boldsymbol{\lambda})$ distribution, as a special case of phase-type distribution, can also be characterized by $(\boldsymbol{\alpha}, \boldsymbol{B})$, where $\boldsymbol{\alpha}=\left(\alpha_{1}, \cdots, \alpha_{n}\right)=$ $(1,0, \cdots, 0)$ is a row vector and $\boldsymbol{B}$ is an $n \times n$ matrix given by:

$$
\boldsymbol{B}=\left(\boldsymbol{b}_{1}^{\top}, \boldsymbol{b}_{2}^{\top}, \cdots, \boldsymbol{b}_{n}^{\top}\right):=\left(\begin{array}{ccccc}
-\lambda_{1} & \lambda_{1} & 0 & \cdots & 0 \\
0 & -\lambda_{2} & \lambda_{2} & \cdots & 0 \\
\vdots & \vdots & \vdots & \vdots & \vdots \\
0 & 0 & 0 & \cdots & -\lambda_{n}
\end{array}\right) .
$$


Here, $\boldsymbol{b}_{i}^{\top}$ denotes the transpose of the row vector $\boldsymbol{b}_{i}$ in a normal sense. Moreover, the random variable $V$ corresponds to the time to absorption of a terminating continuous-time Markov chain $\{I(t), t \geq 0\}$ with state space $\Xi \cup\{0\}=\left\{E_{1}, E_{2}, \cdots, E_{n}, 0\right\}$, initial distribution $\boldsymbol{\alpha}$ and generator $\left(\begin{array}{cc}\boldsymbol{B} & \boldsymbol{b}^{\top} \\ \mathbf{0} & 0\end{array}\right)$, where $\boldsymbol{b}=\left(0, \cdots, 0, \lambda_{n}\right)$ is a row vector of length $n$. We refer to [17] or [18] for more details on phase-type distributions and their properties.

Next, let $\{N(t), t \geq 0\}$ be a renewal process with arriving epochs $\tau_{0}=0<\tau_{1} \leq \cdots \leq \tau_{n-1} \leq$ $\tau_{n} \leq \cdots<\infty$. We call $\{N(t), t \geq 0\}$ a generalized $\operatorname{Erlang}(n, \boldsymbol{\lambda})$ claim-counting process if $V_{i}=$ $\tau_{i}-\tau_{i-1}, i \geq 1$ are independent and generalized $\operatorname{Erlang}(n, \boldsymbol{\lambda})$ distributed. It is noted that each of $V_{i}, i \geq 1$ corresponds to the time to absorption of a terminating continuous-time Markov chain $\left\{I^{(i)}(t), t \geq 0\right\}$. Denote $\{J(t), t \geq 0\}$ to be an underlying state process defined by:

$$
J(t)=I^{(i)}\left(t-\tau_{i-1}\right), \quad \tau_{i-1} \leq t<\tau_{i} .
$$

It follows that $\{J(t), t \geq 0\}$ is a continuous-time Markov chain with state space $\Xi$, initial distribution $\boldsymbol{\alpha}$ and density matrix: $\left(\boldsymbol{b}_{1}^{\top}+\boldsymbol{b}^{\top}, \boldsymbol{b}_{2}^{\top}, \cdots, \boldsymbol{b}_{n}^{\top}\right)$. Typically, $\{N(t), t \geq 0\}$ is also assumed to be a delayed generalized Erlang $(n, \boldsymbol{\lambda})$ claim-counting process, i.e., $V_{1}$ has a generalized Erlang $(i)$ distribution with parameters $\lambda_{n-i+1}, \cdots, \lambda_{n}$. In this case, $\{J(t), t \geq 0\}$ has initial distribution $(0, \cdots, 0,1,0, \cdots, 0)$, with one the $(n-i+1)$-th component. More generally, the underlining initial distribution $\boldsymbol{\alpha}$ can be arbitrarily chosen, such that $\sum_{i=1}^{n} \alpha_{i}=1$; this case will be discussed only in Theorem 3 below.

Now, we introduce a (delayed) generalized $\operatorname{Erlang}(n, \boldsymbol{\lambda})$ risk model given by:

$$
X(t)=X(0)+c t-\sum_{i=1}^{N(t)} X_{i}, \quad t \geq 0
$$

where $X(0) \geq 0$ is the deterministic initial reserve, $c>0$ is the rate of premium income, $\{N(t), t \geq$ $0\}$ is a (delayed) generalized $\operatorname{Erlang}(n, \boldsymbol{\lambda})$ claim-counting process and $\left\{X_{i}, i \geq 1\right\}$ is a sequence of independent and identically distributed positive random variables with common density function $p(x)$, representing the amounts of successive claims. In addition, we assume that $\{N(t), t \geq 0\}$ and $\left\{X_{i}, i \geq 1\right\}$ are independent and further suppose that $c\left(1 / \lambda_{1}+\cdots+1 / \lambda_{n}\right)>\mathbb{E}\left(X_{1}\right)$, assuring ruin is not certain. To emphasize the underlining states of the risk process, we also write $(X(t), J(t)), t \geq 0$ for the (delayed) generalized $\operatorname{Erlang}(n, \boldsymbol{\lambda})$ risk process. In the following, we focus on the risk model where $X(0)=0$. This case usually plays a fundamental role in the derivations; see, e.g., $[1,2,19]$ and $[20]$.

Next, we introduce a dual model of the (delayed) generalized $\operatorname{Erlang}(n, \boldsymbol{\lambda})$ risk model with initial reserve $X(0)=0$. Define:

$$
Y(t)=c t-\sum_{i=1}^{\widetilde{N}(t)} X_{i}, \quad t \geq 0
$$

where $\{\tilde{N}(t), t \geq 0\}$ is a (delayed) generalized $\operatorname{Erlang}(n, \tilde{\boldsymbol{\lambda}})$ claim-counting process with parameter $\widetilde{\boldsymbol{\lambda}}=$ $\left(\lambda_{n}, \cdots, \lambda_{1}\right)$; here "delayed" applies if the first inter-arrival time has a generalized $\operatorname{Erlang}(i)$ distribution with parameters $\lambda_{i}, \cdots, \lambda_{1}$ and $i<n$. Similarly, as above, we construct a underlining state process 
$\{\widetilde{J}(t), t \geq 0\}$ from $\{\widetilde{N}(t), t \geq 0\}$, with state space $\widetilde{\Xi}=\left\{\widetilde{E}_{1}, \widetilde{E}_{2}, \cdots, \widetilde{E}_{n}\right\}:=\left\{E_{n}, E_{n-1}, \cdots, E_{1}\right\}$. Then, $(Y(t), \widetilde{J}(t)), t \geq 0$ consists of a (delayed) generalized $\operatorname{Erlang}(n, \widetilde{\boldsymbol{\lambda}})$ risk process with $Y(0)=0$. For any $t>0$, we define a $t$-dual process $\left\{Y_{t}(s), s \in[0, t]\right\}$ of the generalized $\operatorname{Erlang}(n, \boldsymbol{\lambda})$ risk process $\{X(s), s \geq 0\}$ as follows:

$$
Y_{t}(s)=X(t-)-X(t-s), \quad s \in[0, t] .
$$

Clearly, the $t$-dual process $\left\{Y_{t}(s), s \in[0, t]\right\}$ is left-continuous and has right limits. Denote by $\left\{\overline{Y_{t}}(s), s \in[0, t]\right\}$ a process, modified from $\left\{Y_{t}(s), s \in[0, t]\right\}$, which is right-continuous and has left limits. Obviously, $\left\{\overline{Y_{t}}(s), s \in[0, t]\right\}$ and $\left\{Y_{t}(s), s \in[0, t]\right\}$ follow the same probability law. Furthermore, for any $x>0,\left\{Y_{t}(s), s \in[0, t]\right\}$ conditional on the event $\left(Y_{t}(0)=0, J(t-)=\right.$ $\left.E_{j}, J(0)=E_{i}, Y_{t}(t-)=x\right)$ is the same in law as $\{Y(s), s \in[0, t]\}$ conditional on the event $\left(Y(0)=0, \widetilde{J}(0)=\widetilde{E}_{n-j+1}, \widetilde{J}(t-)=\widetilde{E}_{n-i+1}, Y(t-)=x\right)$. Note that, hereafter, the event $\left(Y_{t}(t-)=x\right)$ can be understood as $\left(Y_{t}(t-) \in[x, x+d x]\right)$ with $d x$ a positive infinitesimal, so does $(Y(t-)=x)$. In this sense, we call $(Y(t), \widetilde{J}(t)), t \geq 0$ the dual model of the risk model $(X(t), J(t)), t \geq 0$.

\section{Results}

Before presenting the results, we introduce the concept of (conditional) measure-preservation. Let $(\Omega, \mathfrak{F}, \mathbb{P})$ be a complete probability space and define two random variables $Z_{1}, Z_{2}$ on it. Without loss of generality, we assume that $Z_{1}$ and $Z_{2}$ have the same image space $(D, \mathfrak{B}(D))$, with $D$ the set of all real functions with right-continuity and left-limits and $\mathfrak{B}(D)$ the set of all the Borel sets in $D$. Denote by $f^{-1}$ the inverse mapping of a mapping $f$. For $A, B \in \mathfrak{B}(D)$, if

$$
\mathbb{P}\left(Z_{1}^{-1}(A)\right)=\mathbb{P}\left(Z_{2}^{-1}(B)\right)
$$

holds, then we say that the set $A$ with respect to $Z_{1}$ and the set $B$ with respect to $Z_{2}$ have measure-preservation. Furthermore, let $A_{1}, B_{1}$ be another two sets in $\mathfrak{B}(D)$, such that $A_{1} \subset A, B_{1} \subset B$. If:

$$
\mathbb{P}\left(Z_{1}^{-1}\left(A_{1}\right) \mid Z_{1}^{-1}(A)\right)=\mathbb{P}\left(Z_{2}^{-1}\left(B_{1}\right) \mid Z_{2}^{-1}(B)\right)
$$

holds, then we say that the set $A_{1}$ conditional on $A$ (with respect to $Z_{1}$ ) and the set $B_{1}$ conditional on $B$ (with respect to $Z_{2}$ ) have measure-preservation. For simplicity, we say that $A_{1}$ and $B_{1}$ are conditional measure-preserving.

Next, define, for any $k \in \mathbb{N}_{0}, t>0, x>0$,

$$
\begin{aligned}
& w_{i, j}(k, t, x)=\mathbb{P}\left(X(s)>0, s \in(0, t) \mid X(0)=0, J(0)=E_{i}, X(t-)=x, J(t-)=E_{j}, N(t-)=k\right), \\
& u_{i, j}(k, t, x)=\mathbb{P}\left(Y(s)<x, s \in(0, t) \mid Y(0)=0, \widetilde{J}(0)=\widetilde{E}_{j}, Y(t-)=x, \widetilde{J}(t-)=\widetilde{E}_{i}, \widetilde{N}(t-)=k\right) .
\end{aligned}
$$

The following result is an immediate consequence of the conditional measure-preservation between the generalized Erlang risk process $(X(t), J(t)), t \geq 0$ and its dual process $(Y(t), \widetilde{J}(t)), t \geq 0$.

Theorem 1. Let $w_{i, j}(k, t, x), u_{i, j}(k, t, x), k \in \mathbb{N}_{0}, t>0, x>0 ; i, j=1, \cdots, n$ be functions defined as above. Then:

$$
w_{i, j}(k, t, x)=u_{n-i+1, n-j+1}(k, t, x), k \in \mathbb{N}_{0}, t>0, x>0 .
$$


Moreover, define, for any $t>0, x>0$,

$$
\begin{aligned}
& \bar{w}_{i, j}(t, x) d x=\mathbb{P}\left(X(s)>0, s \in(0, t), X(t-) \in[x, x+d x], J(t-)=E_{j} \mid X(0)=0, J(0)=E_{i}\right), \\
& \bar{u}_{i, j}(t, x) d x=\mathbb{P}\left(Y(s)<x, s \in(0, t), Y(t-) \in[x, x+d x], \widetilde{J}(t-)=\widetilde{E}_{i} \mid Y(0)=0, \widetilde{J}(0)=\widetilde{E}_{j}\right) .
\end{aligned}
$$

We present below the principal result of this paper.

Theorem 2. Let $\bar{w}_{i, j}(t, x), \bar{u}_{i, j}(t, x), t>0, x>0 ; i, j=1, \cdots, n$ be defined as above. Then:

$$
\lambda_{j} \bar{w}_{i, j}(t, x)=\lambda_{i} \bar{u}_{n-i+1, n-j+1}(t, x), t>0, x>0 .
$$

Our next result gives a generalization of (1) for a certain Erlang risk model.

Theorem 3. Let $\{X(t), t \geq 0\}$ be a generalized Erlang $(n, \boldsymbol{\lambda})$ risk model with $\lambda_{1}=\lambda_{2}=\cdots=\lambda_{n}=$ $\lambda>0$. Assume further that the underlining initial distribution is $\boldsymbol{\alpha}=(1 / n, \cdots, 1 / n)$. If ct $\geq x>0$, then:

$$
\mathbb{P}(X(s)>0, s \in(0, t) \mid X(0)=0, X(t)=x)=\mathbb{P}(X(s)<x, s \in(0, t) \mid X(0)=0, X(t)=x) .
$$

Remark 1. Note that the right-hand side of (4) is closely related to the density of the first hitting time of the Erlang $(n, \boldsymbol{\lambda})$ risk model, which has been discussed in [21]. Under some conditions on the claim sizes and utilizing similar techniques (by inverting the Laplace transform), it is possible to derive some exact formula for (4). Since the calculation is usually technical and it is not the main subject of the paper, we shall omit it.

\section{Discounted Joint Density of the Surplus Prior to Ruin and the Deficit at Ruin}

One of the key quantities in the study of the generalized Erlang $(n, \boldsymbol{\lambda})$ risk model is the discounted joint density of the surplus prior to ruin and the deficit at ruin $f(x, y \mid 0), x, y>0$. It follows from (3.8) and (3.12) in [20] (see also (8.3) in [19]) that:

$$
\begin{aligned}
f(x, y \mid 0) & =\frac{1}{c} \boldsymbol{\alpha}\left(\boldsymbol{V}^{-1} e^{-\Lambda_{\rho} x} \boldsymbol{V}\right) \boldsymbol{b}^{\top} p(x+y) \\
& =\frac{\lambda_{1} \lambda_{2} \cdots \lambda_{n}}{c^{n}} \sum_{j=1}^{n} e^{-\rho_{j} x} \prod_{k=1, k \neq j}^{n} \frac{1}{\rho_{k}-\rho_{j}} p(x+y) \quad x>0, y>0,
\end{aligned}
$$

where $\Lambda_{\rho}=\operatorname{diag}\left(\rho_{1}, \rho_{2}, \cdots, \rho_{n}\right)$ and:

$$
\boldsymbol{V}=\left(\begin{array}{cccc}
\prod_{j=2}^{n} \frac{\lambda_{j}+\delta-c \rho_{1}}{\lambda_{j-1}} & \ldots & \frac{\lambda_{n}+\delta-c \rho_{1}}{\lambda_{n-1}} & 1 \\
\prod_{j=2}^{n} \frac{\lambda_{j}+\delta-c \rho_{2}}{\lambda_{j-1}} & \ldots & \frac{\lambda_{n}+\delta-c \rho_{2}}{\lambda_{n-1}} & 1 \\
\vdots & \ldots & \vdots & \vdots \\
\prod_{j=2}^{n} \frac{\lambda_{j}+\delta-c \rho_{n}}{\lambda_{j-1}} & \cdots & \frac{\lambda_{n}+\delta-c \rho_{n}}{\lambda_{n-1}} & 1
\end{array}\right)
$$

with $\rho_{i}, i=1,2, \cdots, n$ being the roots with positive real parts (assumed to be distinct) of the generalized Lundberg fundamental equation:

$$
\prod_{j=1}^{n}\left(\lambda_{i}+\delta-c s\right)-\lambda_{1} \lambda_{2} \cdots \lambda_{n} \hat{p}(s)=0 .
$$


Here, $\hat{p}(s)=\int_{0}^{\infty} e^{-s x} p(x) d x$. We refer to [20,22,23] for the derivation and the study of the generalized Lundberg fundamental equation.

Further, it is noted from [24,25] that the matrix form of the Laplace transform of the first passage time (to $x$ ) of the generalized $\operatorname{Erlang}(n, \widetilde{\boldsymbol{\lambda}})$ risk process $\{(Y(t), \widetilde{J}(t)), t \geq 0\}$ is given by $\boldsymbol{R}(0 ; x)=$ $\boldsymbol{H} e^{-\Lambda_{\rho} x} \boldsymbol{H}^{-1}$ with:

$$
\boldsymbol{H}=\left(\begin{array}{cccc}
1 & 1 & \cdots & 1 \\
\frac{\lambda_{n}+\delta-c \rho_{1}}{\lambda_{n}} & \frac{\lambda_{n}+\delta-c \rho_{2}}{\lambda_{n}} & \cdots & \frac{\lambda_{n}+\delta-c \rho_{n}}{\lambda_{n}} \\
\vdots & \cdots & \vdots & \vdots \\
\prod_{j=2}^{n} \frac{\lambda_{j}+\delta-c \rho_{1}}{\lambda_{j}} & \prod_{j=2}^{n} \frac{\lambda_{j}+\delta-c \rho_{2}}{\lambda_{j}} & \cdots & \prod_{j=2}^{n} \frac{\lambda_{j}+\delta-c \rho_{n}}{\lambda_{j}}
\end{array}\right) .
$$

Here, by definition:

$$
(\boldsymbol{R}(0 ; x))_{i, j}=\int_{0}^{\infty} e^{-\delta t} \mathbb{P}\left(Y(s)<x, s \in(0, t), Y(t-) \in[x, x+c d t], \widetilde{J}(t-)=\widetilde{E}_{j} \mid Y(0)=0, \widetilde{J}(0)=\widetilde{E}_{i}\right)
$$

Define, for any $x>0$ and any $i, j=1,2, \cdots, n$,

$U_{i, j}(0 ; x)=\int_{0}^{\infty} e^{-\delta t} \mathbb{P}\left(X(s)>0, s \in(0, t), X(t-) \in[x, x+c d t], J(t-)=E_{j} \mid X(0)=0, J(0)=E_{i}\right)$, and denote $\boldsymbol{U}(0 ; x)=\left(U_{i, j}(0 ; x)\right)_{i, j=1}^{n}$.

Theorem 4. With the notation defined above we have, for any $x>0$,

$$
\boldsymbol{V}^{-1} e^{-\Lambda_{\rho} x} \boldsymbol{V}=\boldsymbol{U}(0 ; x)
$$

Remark 2. Note that the matrix equivalence (10) yields a probability expression for the crucial quantity $\boldsymbol{V}^{-1} e^{-\Lambda_{\rho} x} \boldsymbol{V}$ appearing in (5).

Remark 3. Making use of the formula for $\boldsymbol{R}(0 ; x)$, we are able to re-derive the known formula (6), which, in some sense, shows the power of the duality result given in Theorem 2. Specifically, by definition (see, e.g., [20]):

$$
\begin{aligned}
f(x, y \mid 0) d x= & \int_{0}^{\infty} e^{-\delta t} \mathbb{P}\left(X(s)>0, s \in(0, t), X(t-) \in[x, x+d x], J(t-)=E_{n},\right. \\
& \text { there is a ruin-caused claim in } \left.[t, t+d t] \mid X(0)=0, J(0)=E_{1}\right) p(x+y) .
\end{aligned}
$$

Since, further, the event $(\{X(t), t \geq 0\}$ has a ruin-caused claim in $[t, t+d t])$ depending only on the event $\left(J(t-)=E_{n}\right)$ and is independent of the other events occurring before time $t$, we have:

$$
\begin{gathered}
f(x, y \mid 0) d x=\int_{0}^{\infty} e^{-\delta t} \mathbb{P}\left(X(s)>0, s \in(0, t), X(t-) \in[x, x+d x], J(t-)=E_{n}\right. \\
\left.\mid X(0)=0, J(0)=E_{1}\right) \lambda_{n} d t p(x+y) .
\end{gathered}
$$

Substituting $d x$ by $c d t$ and in view of Theorem 2, we obtain:

$$
f(x, y \mid 0)=\int_{0}^{\infty} e^{-\delta t} \frac{\lambda_{1}}{\lambda_{n}} \mathbb{P}\left(Y(s)<x, s \in(0, t), Y(t-) \in[x, x+c d t], \widetilde{J}(t-)=\widetilde{E}_{n}\right.
$$




$$
\begin{gathered}
\left.\mid Y(0)=0, \widetilde{J}(0)=\widetilde{E}_{1}\right) \frac{\lambda_{n}}{c} p(x+y) \\
=(\boldsymbol{R}(0 ; x))_{1, n} \frac{\lambda_{1}}{c} p(x+y) .
\end{gathered}
$$

Moreover, we have, using similar arguments as in [25],

$$
(\boldsymbol{R}(0 ; x))_{1, n}=\frac{\lambda_{2} \cdots \lambda_{n}}{c^{n-1}} \sum_{j=1}^{n} e^{-\rho_{j} x} \prod_{k=1, k \neq j}^{n} \frac{1}{\rho_{k}-\rho_{j}} .
$$

Consequently, the formula in (6) is established by inserting the above into (11).

\section{Proofs}

This section is devoted to the proofs of Theorems 2, 3 and 4 .

We start with a preliminary lemma. Define, for any $k \in \mathbb{N}_{0}, t>0, x>0$,

$$
\begin{array}{r}
\bar{w}_{i, j}(k, t, x) d x=\mathbb{P}\left(X(s)>0, s \in(0, t), X(t-) \in[x, x+d x], J(t-)=E_{j},\right. \\
\left.N(t-)=k \mid X(0)=0, J(0)=E_{i}\right), \\
\bar{u}_{i, j}(k, t, x) d x=\mathbb{P}\left(Y(s)<x, s \in(0, t), Y(t-) \in[x, x+d x], \widetilde{J}(t-)=\widetilde{E}_{i},\right. \\
\left.\widetilde{N}(t-)=k \mid Y(0)=0, \widetilde{J}(0)=\widetilde{E}_{j}\right) .
\end{array}
$$

Lemma 5. Let $\bar{w}_{i, j}(k, t, x), \bar{u}_{i, j}(k, t, x), k \in \mathbb{N}_{0}, t>0, x>0 ; i, j=1, \cdots, n$ be defined as above. Then:

$$
\lambda_{j} \bar{w}_{i, j}(k, t, x)=\lambda_{i} \bar{u}_{n-i+1, n-j+1}(k, t, x), k \in \mathbb{N}_{0}, t>0, x>0 .
$$

Proof: By definition, we have that, conditionally on $\left(J(0)=E_{i}\right)$, the inter-arrival times $V_{i}, i=$ $1,2, \cdots$ can be expressed as:

$$
V_{1}=W_{1, i}+W_{1, i+1}+\cdots+W_{1, n}, \quad V_{k}=W_{k, 1}+W_{k, 2}+\cdots+W_{k, n}, k=2,3, \cdots,
$$

where, for any $i=1,2, \cdots, n,\left\{W_{k, i}\right\}_{k \in \mathbb{N}}$ are independent exponential random variables with common parameter $\lambda_{i}$. Next, let $\mathcal{A}_{k}=\tau_{k}+W_{k+1,1}+W_{k+1,2}+\cdots+W_{k+1, j-1}-W_{1, i}$. It follows that:

$$
\begin{aligned}
& \mathbb{P}\left(X(t-) \in[x, x+d x], J(t-)=E_{j}, N(t-)=k \mid X(0)=0, J(0)=E_{i}\right) \\
& \quad=\mathbb{P}\left(\mathcal{A}_{k}+W_{1, i} \leq t<\mathcal{A}_{k}+W_{1, i}+W_{k+1, j}\right) p^{k *}(c t-x) d x
\end{aligned}
$$

By conditional arguments, direct calculations yield that:

$$
\mathbb{P}\left(\mathcal{A}_{k}+W_{1, i} \leq t<\mathcal{A}_{k}+W_{1, i}+W_{k+1, j}\right)=\frac{\lambda_{i}}{\lambda_{j}-\lambda_{i}} \mathbb{E}\left(e^{-\lambda_{i}\left(t-\mathcal{A}_{k}\right)}-e^{\lambda_{j}\left(t-\mathcal{A}_{k}\right)} \mathbb{I}_{\left(\mathcal{A}_{k} \leq t\right)}\right)
$$

implying thus:

$$
\begin{aligned}
& \mathbb{P}\left(X(t-) \in[x, x+d x], J(t-)=E_{j}, N(t-)=k \mid X(0)=0, J(0)=E_{i}\right) \\
& =\frac{\lambda_{i}}{\lambda_{j}-\lambda_{i}} \mathbb{E}\left(e^{-\lambda_{i}\left(t-\mathcal{A}_{k}\right)}-e^{\lambda_{j}\left(t-\mathcal{A}_{k}\right)} \mathbb{I}_{\left(\mathcal{A}_{k} \leq t\right)}\right) p^{k *}(c t-x) d x
\end{aligned}
$$


Using similar arguments, we also obtain that:

$$
\begin{aligned}
& \mathbb{P}\left(Y(t-) \in[x, x+d x], \widetilde{J}(t-)=\widetilde{E}_{n-i+1}, \widetilde{N}(t-)=k \mid Y(0)=0, \widetilde{J}(0)=\widetilde{E}_{n-j+1}\right) \\
& =\frac{\lambda_{j}}{\lambda_{j}-\lambda_{i}} \mathbb{E}\left(e^{-\lambda_{i}\left(t-\mathcal{B}_{k}\right)}-e^{\lambda_{j}\left(t-\mathcal{B}_{k}\right)} \mathbb{I}_{\left(\mathcal{B}_{k} \leq t\right)}\right) p^{k *}(c t-x) d x
\end{aligned}
$$

where $\mathcal{B}_{k}$ is some random variable, which has the same distribution as $\mathcal{A}_{k}$. Consequently, the claim follows from Theorem 1 and the fact that:

$$
\begin{aligned}
\bar{w}_{i, j}(k, t, x) d x= & \mathbb{P}\left(X(t-) \in[x, x+d x], J(t-)=E_{j}, N(t-)=k \mid X(0)=0, J(0)=E_{i}\right) \\
& \times w_{i, j}(k, t, x), \\
\bar{u}_{i, j}(k, t, x) d x= & \mathbb{P}\left(Y(t-) \in[x, x+d x], \widetilde{J}(t-)=\widetilde{E}_{i}, \widetilde{N}(t-)=k \mid Y(0)=0, \widetilde{J}(0)=\widetilde{E}_{j}\right) \\
& \times u_{i, j}(k, t, x) .
\end{aligned}
$$

This completes the proof.

Proof of Theorem 2: The claim follows by taking the sum from $k=0$ to $k=\infty$ in (12).

Proof of Theorem 3: First, note that:

$$
\begin{aligned}
& \mathbb{P}(X(s)>0, s \in(0, t) \mid X(0)=0, X(t)=x) \\
& \quad=\frac{\mathbb{P}(X(s)>0, s \in(0, t), X(t) \in[x, x+d x] \mid X(0)=0)}{\mathbb{P}(X(t) \in[x, x+d x] \mid X(0)=0)} .
\end{aligned}
$$

Since $\lambda_{1}=\lambda_{2}=\cdots=\lambda_{n}=\lambda$, both $(X(t), J(t)), t \geq 0$ and $(X(t), \widetilde{J}(t)), t \geq 0$ are generalized Erlang risk models with the underlining initial distribution $(1 / n, \cdots, 1 / n)$, but with different (only in notation) underlining states $\Xi$ and $\widetilde{\Xi}$. Furthermore, we have that the dual process $(Y(t), \widetilde{J}(t)), t \geq 0$ of $(X(t), J(t)), t \geq 0$ follows the same conditional probability law as $(X(t), \widetilde{J}(t)), t \geq 0$, given that the initial underling state is known (e.g., given $\widetilde{J}(0)=\widetilde{E}_{i}$ ). In view of Theorem 2:

$$
\bar{w}_{i, j}(t, x)=\bar{u}_{n-i+1, n-j+1}(t, x)
$$

holds for any $t>0, x>0$. Therefore, by noting that $\mathbb{P}(X(t) \neq X(t-))=0$, we conclude that:

$$
\begin{aligned}
& \mathbb{P}(X(s)>0, s \in(0, t), X(t) \in[x, x+d x] \mid X(0)=0) \\
& =\sum_{i=1}^{n} \sum_{j=1}^{n} \mathbb{P}\left(J(0)=E_{i} \mid X(0)=0\right) \bar{w}_{i, j}(t, x) d x=\sum_{i=1}^{n} \sum_{j=1}^{n} \frac{1}{n} \bar{w}_{i, j}(t, x) d x \\
& =\sum_{i=1}^{n} \sum_{j=1}^{n} \mathbb{P}\left(\widetilde{J}(0)=\widetilde{E}_{n-j+1} \mid X(0)=0\right) \bar{u}_{n-i+1, n-j+1}(t, x) d x \\
& =\mathbb{P}(X(s)<x, s \in(0, t), X(t) \in[x, x+d x] \mid X(0)=0) .
\end{aligned}
$$

Consequently, the claim follows by inserting the above formula into (13). This completes the proof.

Proof of Theorem 4: Denote by $A^{T}$ the transpose of an $n \times n$ matrix $A$ with respect to the counter-diagonal, namely, $\left(A^{T}\right)_{i, j}=A_{n-j+1, n-i+1}$. Elementary calculations show that, for any $n \times n$ matrices $A$ and $B$,

$$
(A B)^{T}=B^{T} A^{T},\left(A^{T}\right)^{T}=A, \quad\left(A^{-1}\right)^{T}=\left(A^{T}\right)^{-1} .
$$


Thus,

$$
\left(\boldsymbol{V}^{-1} e^{-\Lambda_{\rho} x} \boldsymbol{V}\right)^{T}=\boldsymbol{V}^{T}\left(e^{-\Lambda_{\rho} x}\right)^{T}\left(\boldsymbol{V}^{-1}\right)^{T}
$$

Furthermore, in view of (7) and (9), direct calculations yield that:

$$
\left(\boldsymbol{V}^{T}\right)_{i, j}=\frac{\lambda_{n}}{\lambda_{n-i+1}} \boldsymbol{H}_{i, n-j+1}, \quad\left(\left(\boldsymbol{V}^{-1}\right)^{T}\right)_{i, j}=\frac{\lambda_{n-j+1}}{\lambda_{n}}\left(\boldsymbol{H}^{-1}\right)_{n-i+1, j} .
$$

Clearly, $\left(e^{-\Lambda_{\rho} x}\right)^{T}=\operatorname{diag}\left(e^{-\rho_{n} x}, e^{-\rho_{n-1} x}, \cdots, e^{-\rho_{1} x}\right)$. Therefore,

$$
\begin{aligned}
\left(\left(\boldsymbol{V}^{-1} e^{-\Lambda_{\rho} x} \boldsymbol{V}\right)^{T}\right)_{i, j} & =\frac{\lambda_{n-j+1}}{\lambda_{n-i+1}}\left(\boldsymbol{H}_{i, n}, \cdots, \boldsymbol{H}_{i, 1}\right)\left(e^{-\Lambda_{\rho} x}\right)^{T}\left(\left(\boldsymbol{H}^{-1}\right)_{n, j}, \cdots,\left(\boldsymbol{H}^{-1}\right)_{1, j}\right)^{\top} \\
& =\frac{\lambda_{n-j+1}}{\lambda_{n-i+1}} \sum_{k=1}^{n} \boldsymbol{H}_{i, k} e^{-\rho_{k} x}\left(\boldsymbol{H}^{-1}\right)_{k, j}=\frac{\lambda_{n-j+1}}{\lambda_{n-i+1}}\left(\boldsymbol{H} e^{-\Lambda_{\rho} x} \boldsymbol{H}^{-1}\right)_{i, j} \\
& =\frac{\lambda_{n-j+1}}{\lambda_{n-i+1}}(\boldsymbol{R}(0 ; x))_{i, j} .
\end{aligned}
$$

Consequently, we conclude from Theorem 2 that:

$$
\begin{aligned}
\left(\boldsymbol{V}^{-1} e^{-\Lambda_{\rho} x} \boldsymbol{V}\right)_{i, j} & =\frac{\lambda_{i}}{\lambda_{j}}(\boldsymbol{R}(0 ; x))_{n-j+1, n-i+1} \\
& =U_{i, j}(0 ; x)
\end{aligned}
$$

implying (10), and thus the proof is complete.

\section{Acknowledgments}

We are in debt to two referees for numerous comments and suggestions. Both authors kindly acknowledge partial support by the Swiss National Science Foundation Project 200021-140633/1 and the FP7 Grant PIRSES-GA-2012-318984. C. Zhang also acknowledges partial support by the National Science Foundation of China (Grant Nos. 11171164, 11271385 and 11371020).

\section{Author Contributions}

The ideas and results of this paper came from many discussions between L. Ji and C. Zhang; both of them contributed to the proofs and the writing.

\section{Conflicts of Interest}

The authors declare no conflict of interest.

\section{References}

1. Gerber, H. U.; Shiu, E. S. W. The joint distribution of the time of ruin, the surplus immediately before ruin, and the deficit at ruin. Insur. Math. Econom. 1997, 21, 129-137. 
2. Gerber, H. U.; Shiu, E. S. W. On the time value of ruin. N. Am. Actuar. J. 1998, 2 , 48-78.

3. Dickson, D. C. M. On the distribution of surplus prior to ruin. Insur. Math. Econom. 1992, 11, 191-207.

4. Dickson, D. C. M.; Egídio Dos Reis, A. D. Ruin problems and dual events. Insur. Math. Econom. 1994, 14, 51-60.

5. Feller, W. An Introduction to Probability Theory and Its Applications; Willey: Now York, NY, USA, 1971.

6. Gerber, H. U. Mathematical fun with ruin theory. Insur. Math. Econom. 1988, 7, 15-23.

7. Lefèvre, C.; Loisel, S. On finite-time ruin probabilities for classical risk models. Scand. Actuar. J. 2008, 1, 41-60.

8. Lefèvre, C.; Picard, P. Ruin time and severity for a Lévy subordinator claim process: A simple approach. Risks 2013, 1, 192-212.

9. Borovkov, K. A.; Dickson, D. C. M. On the ruin time distribution for a Sparre Andersen process with exponential claim sizes. Insur. Math. Econom. 2008, 42, 1104-1108.

10. Zhao, C.; Zhang, C. Joint density of the number of claims until ruin and the time to ruin in the delayed renewal risk model with $\operatorname{Erlang}(n)$ claims. J. Comput. Appl. Math. 2013, 244, 102-114.

11. Albrecher, H.; Boxma, O.; Ivanovs, J. On simple ruin expressions in dependent Sparre Andersen risk models. J. Appl. Probab. 2014, 51, 293-296.

12. Asmussen, S.; Højgaard, B. Finite horizon ruin probabilities for Markov-modulated risk processes with heavy tails. Theory Stoch. Process. 1996, 2, 96-107.

13. Liu, P.; Zhang, C.; Ji, L. Joint densities of the time to ruin and the number of claims until ruin in perturbed classical risk models. 2014, preprint.

14. Miyazama, M. Hitting probabilities in a Markov additive process with linear movements and upward jumps: Applications to risk and queueing processes. Ann. Appl. Probab. 2004, 14, 1029-1054.

15. Dickson, D. C. M.; Li, S. Erlang risk models and finite time ruin problems. Scand. Actuar. J. 2012, 3, 183-202.

16. Lefèvre, C.; Picard, P. Appell pseudopolynomials and Erlang-type risk models. Stoch. Int. J. Probab. Stoch. Process. 2014, 86, 676-695.

17. Asmussen, S.; Albrecher, H. Ruin Probabilities; World Scientific: Hackensack, NJ, USA, 2010.

18. Rolski, T.; Schmidli, H.; Schmidt, V.; Teugels, J. Stochastic processes for insurance and finance. Wiley Series in Probability and Statistics. John Wiley \& Sons Ltd., Chichester, 1999.

19. Gerber, H. U.; Shiu, E. S. W. On the time value of ruin in a Sparre Andersen model. N. Am. Actuar. J. 2005, 9, 49-69.

20. Ren, J. The discounted joint distribution of the surplus prior to ruin and the deficit at ruin in a Sparre Andersen model. N. Am. Actuar. J. 2007, 11, 128-136.

21. Dickson, D. C. M.; Li, S. The distributions of the time to reach a given level and the duration of negative surplus in the Erlang(2) risk model. Insur. Math. Econom. 2013, 52, 490-497.

22. Bergel, A. I.; Egidio dos Reis, A. D. On a Sparre-Andersen risk model with PH(n) interclaim times. Preprint, 2014. Available Online: http://cemapre.iseg.ulisboa.pt/archive/preprints/617.pdf (accessed on 28 February 2013). 
23. Ji, L.; Zhang, C. Analysis of the multiple roots of the Lundberg fundamental equation in the $\mathrm{PH}(n)$ risk model. Appl. Stoch. Models Bus. Ind. 2012, 28, 73-90.

24. $\mathrm{Li}, \mathrm{S}$. Discussion of "The discounted joint distribution of the surplus prior to ruin and the deficit at ruin in a Sparre Andersen model". N. Am. Actuar. J. 2008, 12, 208-210.

25. Li, S. The time of recovery and the maximum severity of ruin in a Sparre Andersen model". $N$. Am. Actuar. J. 2008, 12, 413-425.

(C) 2014 by the authors; licensee MDPI, Basel, Switzerland. This article is an open access article distributed under the terms and conditions of the Creative Commons Attribution license (http://creativecommons.org/licenses/by/4.0/). 